\title{
Deformation of singular lagrangian subvarieties
}

\author{
Duco van Straten and Christian Sevenheck
}

February 10, 2000

\begin{abstract}
We investigate deformations of lagrangian manifolds with singularities. We introduce a complex similar to the de Rham-complex whose cohomology calculates deformation spaces. Examples of singular lagrangian varieties are presented and deformations are calculated explicitly.
\end{abstract}

\section{Introduction}

In this paper, we develop some ideas of a deformation theory of singular lagrangian subvarieties. Lagrangian submanifolds are quite fundamental objects, so in a sense it is natural to extend the study of them to a larger class of objects which are allowed to have singularities. This has been done by Arnold, Givental and others (|Giv88 $\mid)$. However, not much is known on the behavior of lagrangian singularities under deformations. The aim of this article is to describe the spaces of infinitesimal deformations and obstructions of a lagrangian subvariety and to perform calculations for some concrete examples. It turns out that the lagrangian property of a space has a strong influence on its deformations, e.g., there are examples of spaces $X$ with $\operatorname{dim}\left(T_{X}^{1}\right)=\infty$, which have nevertheless a versal deformation space for the lagrangian deformations.

In the sequel, we will consider the following situation: Let $M$ be a $2 n$ dimensional symplectic manifold over $\mathbb{K}=\mathbb{R}$ or $\mathbb{K}=\mathbb{C}$ (that is, a $C^{\infty}$ or complex analytic manifold of real resp. complex dimension $2 n$ endowed with a closed, non-degenerated 2-form $\omega$, holomorphic in the second case) and $L$ a reduced analytic subspace of dimension $n$, given by an involutive ideal sheaf $\mathcal{I}$, i.e. an ideal sheaf satisfying $\{\mathcal{I}, \mathcal{I}\} \subset \mathcal{I}$ where $\{$,$\} denotes the Poisson bracket$ corresponding to $\omega$. This condition ensures that $L$ is a lagrangian submanifold in a neighborhood of each of its smooth points. A lagrangian deformation of $L$ will be a deformation in the usual sense (a flat family $L_{S} \rightarrow S$ ) with the additional condition that all fibers are lagrangian subvarieties of $M$. More precisely, we

1991 Mathematics Subject Classification. Primary 14B05, 14B12, 58F05; Secondary $32 \mathrm{~S} 40,32 \mathrm{~S} 60$ 
will call a diagram

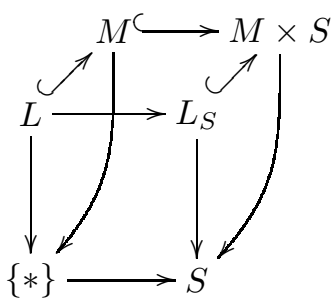

a lagrangian deformation of $L$ iff $L_{S} \rightarrow S$ is flat and $\left.\left\{\mathcal{I}_{S}, \mathcal{I}_{S}\right\}\right\}_{S} \subset \mathcal{I}_{S}$. Here $\mathcal{I}_{S}$ is the ideal sheaf defining $L_{S}$ in $M \times S$ and $\{,\}_{S}$ is the Poisson structure defined on $M \times S$ by the (degenerate) form $\omega_{S}:=p^{*} \omega, p: M \times S \rightarrow M$ being the canonical projection. This definition can be formalized using the language of deformation functors (see Sev99] and Sch68). This more formal approach yields the definition of morphisms of deformations, in particular, two deformations $L_{S} \subset M \times S$ and $L_{T}^{\prime} \subset N \times T$ are called equivalent iff there is an fibrewise isomorphism $F: M \times S \rightarrow N \times T$ satisfying $F^{*} \omega_{T}=\omega_{S}$. Such an $F$ comes from a symplectic diffeomorphism $f: M \rightarrow N$ and in case that $M$ is simply connected (which we will suppose from now on), $f$ is induced by an hamiltonian vector field, see lemma 3.

The tangent space to the functor of lagrangian deformations of $L$ (that is, the space of lagrangian deformations of $L$ over $\operatorname{Spec}(\mathbb{K}[\epsilon])$ up to those induced by hamiltonian vector fields of the ambient manifold) will be denoted by $L T_{L}^{1}$. However, we will focus our attention to the local case mainly, that is, we will study the sheaf $\mathcal{L T}_{L}^{1}$ of lagrangian deformations of $L$. For lagrangian submanifolds, it follows from |Voi92], that the versal deformation space is smooth, i.e., deformation of such objects are unobstructed. This is probably not true in the singular case, although an example has not been found yet. See theorem 1 for further details.

Acknowledgements: We would like to thank A. Givental who suggested to investigate the deformation theory of lagrangian singularities in december 1992.

\section{The complex $\mathcal{C}^{\bullet}$}

We start with a slightly more general situation: Let $\mathcal{I} \subset \mathcal{O}_{M}$ be an involutive ideal sheaf, $\mathcal{O}_{L}$ the structure sheaf of the subvariety $L$ described by $\mathcal{I}$ and denote by $\mathcal{L}:=\mathcal{I} / \mathcal{I}^{2}$ the conormal sheaf. The formula $\left\{\mathcal{I}^{i}, \mathcal{I}^{j}\right\} \subset \mathcal{I}^{i+j-1}$, which can be easily verified, shows that there are well-defined operations

$$
\begin{aligned}
& \mathcal{L} \times \mathcal{O}_{L} \quad \longrightarrow \mathcal{O}_{L} \quad \text { and } \quad \mathcal{L} \times \mathcal{L} \longrightarrow \mathcal{L} \\
& (g, f) \longmapsto\{g, f\} \quad \text { and } \quad(g, h) \longmapsto\{g, h\}
\end{aligned}
$$

compatible in the sense that $\{g, f \cdot h\}=\{g, f\} h+f\{g, h\}$. This implies that the first operation can be rewritten as a morphism

$$
\mathcal{L} \rightarrow \operatorname{Der}\left(\mathcal{O}_{L}, \mathcal{O}_{L}\right)=\Theta_{L}
$$

One says that $\mathcal{L}$ is a Lie algebroid (for details on Lie algebroids, see Mac87]). 
Definition 1. Let $\mathcal{C}_{L}^{p}$ be the following $\mathcal{O}_{L}$-module

$$
\mathcal{C}_{L}^{p}:=\mathcal{H o m}_{\mathcal{O}_{L}}\left(\bigwedge^{p} \mathcal{L}, \mathcal{O}_{L}\right)
$$

and define a differential:

$$
\begin{aligned}
& (\delta(\phi))\left(h_{1} \wedge \ldots \wedge h_{p+1}\right):= \\
& \sum_{i=1}^{p+1}(-1)^{i}\left\{h_{i}, \phi\left(h_{1} \wedge \ldots \wedge \widehat{h}_{i} \wedge \ldots h_{p+1}\right)\right\} \\
& +\sum_{1 \leq i<j \leq p+1}(-1)^{i+j-1} \phi\left(\left\{h_{i}, h_{j}\right\} \wedge h_{1} \wedge \ldots \wedge \widehat{h}_{i} \wedge \ldots \wedge \widehat{h}_{j} \wedge \ldots \wedge h_{p+1}\right)
\end{aligned}
$$

It is a straightforward computation to check that $\delta \circ \delta=0$, so we get indeed a complex. Following Mac87, it is called the standard complex for the Lie algebroid $\mathcal{L}$. Remark that $\mathcal{C}^{0}=\mathcal{O}_{L}$ and $\mathcal{C}^{1}=\mathcal{H o m}_{\mathcal{O}_{L}}\left(\mathcal{I} / \mathcal{I}^{2}, \mathcal{O}_{L}\right)=: \mathcal{N}_{L}$, the normal sheaf of $\mathcal{I}$ in $\mathcal{O}_{M}$. For the definition of $\delta$, the fact that $\mathcal{I}$ is involutive is essential: the second term would not make sense otherwise.

We may define a product on the complex $\left(\mathcal{C}^{\bullet}, \delta\right)$ :

$$
\begin{aligned}
\mathcal{C}^{p} \times \mathcal{C}^{q} & \longrightarrow \mathcal{C}^{p+q} \\
(\Phi, \Psi) & \longmapsto \Phi \wedge \Psi
\end{aligned}
$$

with

$$
\begin{aligned}
& (\Phi \wedge \Psi)\left(f_{1} \wedge \ldots \wedge f_{p+q}\right)= \\
& \sum_{\substack{I \amalg J=\{1, \ldots, n\} \\
i_{1}<\ldots<i_{p} \\
j_{1}<\ldots<j_{q}}} \operatorname{sgn}(I, J) \cdot \Phi\left(f_{i_{1}} \wedge \ldots \wedge f_{i_{p}}\right) \cdot \Psi\left(f_{j_{1}} \wedge \ldots \wedge f_{j_{q}}\right) \\
&
\end{aligned}
$$

The sign is defined as

$$
\operatorname{sgn}(I, J):=\operatorname{sgn}\left(\begin{array}{l}
1, \ldots \ldots \ldots, p+q \\
i_{1}, \ldots, i_{p}, j_{1}, \ldots, j_{q}
\end{array}\right)
$$

Proposition 1. Let $\Phi \in \mathcal{C}^{p}, \Psi \in \mathcal{C}^{q}$ et $\Gamma \in \mathcal{C}^{r}$. Then we have

1. $\Phi \wedge \Psi=(-1)^{\operatorname{deg}(\Phi) \cdot \operatorname{deg}(\Psi)} \cdot \Psi \wedge \Phi$

2. $(\Phi \wedge \Psi) \wedge \Gamma=\Phi \wedge(\Psi \wedge \Gamma)$

3. $\delta(\Phi \wedge \Psi)=\delta(\Phi) \wedge \Psi+(-1)^{\operatorname{deg}(\Phi)} \cdot \Phi \wedge \delta(\Psi)$

Proof. The first two points are trivial, while the third has to be checked by an explicit calculation.

Note that the last proposition says that $\left(\mathcal{C}_{L}^{\bullet}, \delta, \wedge\right)$ is a differential graded algebra, furthermore, we have $\mathcal{C}_{L}^{0}=\mathcal{O}_{L}=\Omega_{L}^{0}$. As one might hope, there is indeed a tight connection between $\Omega_{L}^{\bullet}$ and $\mathcal{C}_{L}^{\bullet}$.

Proposition 2. Suppose that $L$ is lagrangian. Then there exists a morphism $J: \Omega_{L}^{1} \rightarrow \mathcal{C}_{L}^{1}$ which is an isomorphism outside the singular locus of $L$. 
Proof. On a symplectic manifold, there is a canonical isomorphism $\beta$ between vector fields and one forms, given by $\beta(V):=i_{V} \omega$. On the other hand, for each analytic subspace $L \subset M$ we have two exact sequences, dual to each other, namely, the conormal and the normal sequence, thus, there is the following diagram:

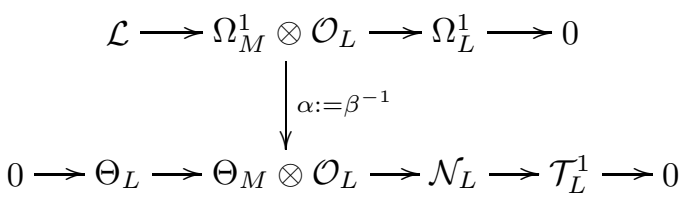

Now the fundamental fact is that this diagram can be completed: the morphism $\mathcal{L} \rightarrow \Theta_{L}$ above commutes with $\alpha$, so we have

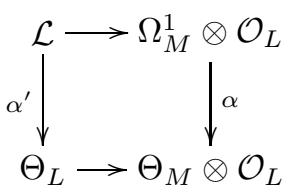

Note that the image of an element $g \in \mathcal{L}$ under $\alpha^{\prime}$ is just the hamiltonian vector field $H_{g}$. The morphisms $J: \Omega_{L}^{1} \rightarrow \mathcal{C}_{L}^{1}=\mathcal{N}_{L}$ we are looking for can now be defined as the map induced by $\alpha$, explicitly

$$
J(d f)=(g \mapsto\{f, g\})
$$

To see that $J$ is an isomorphism near a smooth point of $L$ it will be sufficient to prove this for the map $\alpha^{\prime}$ (because at smooth points $x$ we have $\mathcal{T}_{(L, x)}^{1}=0$ and the map $\mathcal{L}_{x} \rightarrow \Omega_{(L, x)}^{1} \otimes \mathcal{O}_{L, x}$ is injective). So assume the sheaves $\mathcal{L}, \Omega_{L}^{1}$, and $\Theta_{L}$ to be defined in a neighborhood of a smooth point which means that they all become locally free. $\mathcal{L}$ then has to be identified with the conormal bundle. To prove that $\alpha^{\prime}$ is an isomorphism, we will construct an inverse. First note that, by the fact that $L$ is coisotropic, the morphism $\beta: \Theta_{M \mid L} \rightarrow \Omega_{M \mid L}^{1}$ actually sends an element of $\Theta_{L}$ to a form vanishing on all vectors tangent to $L$. So the restriction of $\beta$ to $\Theta_{L}$ defines a morphism $\beta^{\prime}: \Theta_{L} \rightarrow \mathcal{L}$. The situation is as follows:

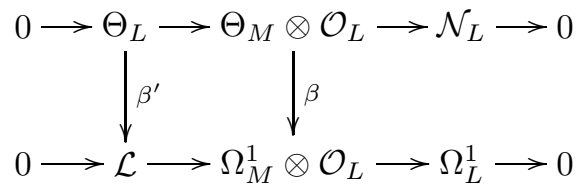

A diagram chase shows that $\beta^{\prime}$ is injective. On the other hand, we have $\operatorname{dim}(\mathcal{L})=\operatorname{dim}\left(\Theta_{L}\right)$, as $L$ is lagrangian. So $\beta^{\prime}$ is an isomorphism and the inverse of $\alpha^{\prime}$.

Corollary 1. The morphism $J: \Omega_{L}^{1} \rightarrow \mathcal{C}_{L}^{1}$ can be extended to a morphism of $D G A$ 's

$$
J:\left(\Omega_{L}^{\bullet}, d, \wedge\right) \longrightarrow\left(\mathcal{C}_{L}^{\bullet}, \delta, \wedge\right)
$$

which is an isomorphism at smooth points of $L$. 
Proof. Set

$$
J\left(\omega_{1} \wedge \ldots \wedge \omega_{p}\right):=J\left(\omega_{1}\right) \wedge \ldots \wedge J\left(\omega_{p}\right)
$$

where $\omega_{i} \in \Omega_{L}^{1}$. Then it is immediate that $J$ is an isomorphism on $L_{r e g}$. To prove that $J \circ d=\delta \circ J$, it suffices to check this in the lowest degrees, that is, we have to show that the diagram

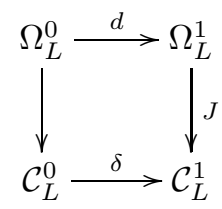

commutes. This follows directly from $\Omega_{L}^{0}=\mathcal{C}_{L}^{0}=\mathcal{O}_{L}$.

In the last section, we use the following elementary fact.

Lemma 1. The kernel of $J$ is the complex $\mathcal{T o r s}\left(\Omega_{L}^{\bullet}\right)$ consisting of the torsion subsheaves of $\Omega_{L}^{p}$.

Proof. We have $\operatorname{Tors}\left(\Omega_{L}^{\bullet}\right) \subset \operatorname{Ker}(J)$ as $\mathcal{C}_{L}^{\bullet}$ is torsion free. On the other hand, the kernel is supported on the singular locus of $L$, so it must be a torsion sheaf, hence $\operatorname{Ker}(J) \subset \mathcal{T} \operatorname{Tors}\left(\Omega_{L}^{\bullet}\right)$.

Remark: Although the definition of the modules $\mathcal{C}_{L}^{p}$ involves the ideal $\mathcal{I}$, they are probably intrinsic. This is at least clear in some special cases as the following lemma shows.

Lemma 2. Suppose $L$ to be Cohen-Macaulay and regular in codimension one. Then there is an isomorphism

$$
\left(\Omega_{L}^{p}\right)^{* *} \stackrel{\cong}{\longrightarrow} \mathcal{C}_{L}^{p}
$$

where for an $\mathcal{O}_{L}$-module $\mathcal{F}, \mathcal{F}^{*}$ denotes $\mathcal{H o m}_{\mathcal{O}_{L}}\left(\mathcal{F}, \mathcal{O}_{L}\right)$.

Proof. We will make use of the following fact: Let $\mathcal{F}$ be an $\mathcal{O}_{L}$-module of type $\mathcal{G}^{*}$, then $\mathcal{F}$ is reflexive, i.e. $\mathcal{F}^{* *}=\mathcal{F}$. The morphism $h:\left(\Omega_{L}^{p}\right)^{* *} \rightarrow \mathcal{C}_{L}^{p}$ we are looking is obtained by dualizing twice the morphism $J: \Omega_{L}^{p} \rightarrow \mathcal{C}_{L}^{p}$, this yields $J^{* *}:\left(\Omega_{L}^{p}\right)^{* *} \rightarrow\left(\mathcal{C}_{L}^{p}\right)^{* *}=\mathcal{C}_{L}^{p}$ as $\mathcal{C}_{L}^{p}$ is of type $\mathcal{H o m}\left(-, \mathcal{O}_{L}\right)$. Clearly, $h$ is an isomorphism on the regular locus. We have an exact sequence

$$
0 \longrightarrow \mathcal{K} \longrightarrow\left(\Omega_{L}^{p}\right)^{* *} \stackrel{h}{\longrightarrow} \mathcal{C}_{L}^{p} \longrightarrow \mathcal{G} \longrightarrow 0
$$

where $\mathcal{K}$ and $\mathcal{G}$ are the kernel resp. cokernel sheaves of the map $h$. This sequence can be split

$$
\begin{gathered}
0 \longrightarrow \mathcal{K} \longrightarrow\left(\Omega_{L}^{p}\right)^{* *} \longrightarrow \mathcal{H} \longrightarrow 0 \\
0 \longrightarrow \mathcal{H} \longrightarrow \mathcal{C}_{L}^{p} \longrightarrow \mathcal{G} \longrightarrow 0
\end{gathered}
$$

with $\mathcal{H}=\operatorname{Im}(h)$. Applying $\mathcal{H o m}_{\mathcal{O}_{L}}\left(-, \mathcal{O}_{L}\right)$ yields

$$
\begin{aligned}
& 0 \longrightarrow \mathcal{H}^{*} \longrightarrow\left(\left(\Omega_{L}^{p}\right)^{* *}\right)^{*} \longrightarrow \mathcal{K}^{*} \\
& 0 \longrightarrow \mathcal{G}^{*} \longrightarrow\left(\mathcal{C}_{L}^{p}\right)^{*} \stackrel{\mathcal{H}^{*}}{\longrightarrow} x t^{1}\left(\mathcal{G}, \mathcal{O}_{L}\right)
\end{aligned}
$$

Now we use the lemma of Ischebeck (see Mat89]): Given a local ring $R$, two $R$ modules $M$ and $N$ with $k=\operatorname{dim}(M)$ and $r=\operatorname{depth}(N)$, then for all $p<r-k$, the modules $\operatorname{Ext}^{p}(M, N)$ vanish. It follows that $\mathcal{K}^{*}=\mathcal{G}^{*}=\mathcal{E} x t^{1}\left(\mathcal{G}, \mathcal{O}_{L}\right)=0$, so we have $\left(\left(\Omega_{L}^{p}\right)^{* *}\right)^{*}=\left(\mathcal{C}_{L}^{p}\right)^{*}$. Then obviously $\left(\left(\Omega_{L}^{1}\right)^{* *}\right)^{* *}=\left(\mathcal{C}_{L}^{1}\right)^{* *}$ and by the argument above $\left(\Omega_{L}^{1}\right)^{* *}=\mathcal{C}_{L}^{1}$ so the map $h$ is an isomorphism. 


\section{Deformations}

Recall that the space of infinitesimal embedded deformations of an analytic algebra $R$, given as $R=S / I$ where $S$ is the ring of convergent power series, is equal to the normal module of $I$ in $S$, i.e. $\operatorname{Hom}_{R}\left(I / I^{2}, R\right)$. Dividing out trivial deformations gives the space $T_{R}^{1}$, defined by the sequence

$$
0 \longrightarrow \operatorname{Hom}_{R}\left(\Omega_{R}^{1}, R\right) \rightarrow \operatorname{Hom}_{S}\left(\Omega_{S}^{1}, S\right) \widehat{\otimes} R \rightarrow H_{R}\left(I / I^{2}, R\right) \longrightarrow T_{R}^{1} \longrightarrow 0
$$

On the other hand, the deformations of a manifold $X$ over $\operatorname{Spec}\left(\mathbb{K}[\epsilon] /\left(\epsilon^{2}\right)\right)$ are parameterized by $H^{1}\left(X, \Theta_{X}\right)$. The cotangent complex is a tool to handle these two special cases in an integrated manner: infinitesimal deformations of an analytic space $L$ are in bijection with $\mathbb{H}^{1}\left(\mathbb{L}_{X}\right)$. It seems that the complex $\mathcal{C}_{L}^{\bullet}$ has to be seen as a first approximation to an equivalent for the cotangent complex in the lagrangian context. More precisely, the following holds:

Theorem 1. The first three cohomology sheaves of $\mathcal{C}_{L}^{\bullet}$ are

- $\mathcal{H}^{0}\left(\mathcal{C}_{L}^{\bullet}\right)=\mathbb{K}_{L}$.

- $\mathcal{H}^{1}\left(\mathcal{C}_{L}^{\bullet}\right)=\mathcal{L T}_{L}^{1}$.

- $\mathcal{H}^{2}\left(\mathcal{C}_{L}^{\bullet}\right)=\mathcal{L T}_{L}^{2}$. This symbol denotes the lagrangian obstructions, that is, $\mathcal{L T}_{L}^{2}$ is the sheaf of obstructions to extend a lagrangian deformation to higher order regardless whether it can be extended as a flat deformation.

The proof of the following preliminary lemma can be found in [Ban94].

Lemma 3. If $H^{1}(M, \mathbb{K})=0$, then each diffeomorphism $f: M \rightarrow M$ satisfying $f^{*} \omega=\omega$ is the time 1 map of a flow $\varphi_{t}$ of a hamiltonian vector field $H_{g}$ for some function $g$ on $M$.

Proof of the theorem. $\mathcal{H}^{0}\left(\mathcal{C}_{L}^{\bullet}\right)$ equals $\operatorname{Ker}\left(\delta: \mathcal{O}_{L} \rightarrow \mathcal{C}_{L}^{1}\right)$. Take an element $f$ of $\operatorname{Ker}(\delta)$. Then $\{f, g\} \in \mathcal{I}$ for all $g \in \mathcal{I}$. If $f$ is not a constant, then the ideal $(\mathcal{I}, f)$ is strictly larger than $\mathcal{I}$, not the whole ring and still involutive. This is a contradiction to the fact that $L$ is lagrangian, which means that $\mathcal{I}$ is maximal under all involutive ideals. So the kernel must be the constant sheaf.

To prove that $\mathcal{H}^{1}\left(\mathcal{C}_{L}^{\bullet}\right)=\mathcal{L T}_{L}^{1}$, two things have to be checked: As $\mathcal{C}_{L}^{1}=\mathcal{N}_{L}$, we must first identify the elements of $\operatorname{Ker}\left(\delta^{1}: \mathcal{C}_{L}^{1} \rightarrow \mathcal{C}_{L}^{2}\right)$ with the flat lagrangian deformations. Then we have to show that the image of $\delta^{0}: \mathcal{O}_{L} \rightarrow \mathcal{C}_{L}^{1}$ are the trivial deformations. But this is easy, because for $f \in \mathcal{O}_{L}, \delta(f)$ acts as $H_{f}$, thus inducing a trivial deformation. Furthermore, by lemma 3, of all deformations coming from vector fields on $M$, only those induced by hamiltonian vector fields are trivial in the lagrangian sense. Now we choose an open set $U \subset L$ and sections $\left(f_{1}, \ldots, f_{k}\right)$ generating $\mathcal{I}(U)$. Take an element $\Phi \in \operatorname{Ker}\left(\delta^{1}\right)$, which means that

$$
\phi(\{g, h\})-\{g, \phi(h)\}-\{\phi(g), h\}=0
$$

for all $f, g \in \mathcal{I} / \mathcal{I}^{2}$. Then $\Phi$ corresponds to the deformation given by

$$
\widetilde{\mathcal{I}}=\left(f_{1}+\epsilon \phi\left(f_{1}\right), \ldots, f_{k}+\epsilon \phi\left(f_{k}\right)\right)
$$


The ideal $\widetilde{\mathcal{I}}$ is involutive iff for any two elements $f+\epsilon \phi(f), g+\epsilon \phi(g)$, we have $\{f+\epsilon \phi(f), g+\epsilon \phi(g)\} \in \widetilde{\mathcal{I}}$, which is equivalent to

$$
F:=\{f, g\}+\epsilon(\{f, \phi(g)\}+\{\phi(f), g\}) \in \widetilde{\mathcal{I}}
$$

Consider $G:=\{f, g\}+\epsilon \phi(\{f, g\})$, which is an element of $\widetilde{\mathcal{I}}$, so the condition $F \in \widetilde{\mathcal{I}}$ is equivalent to $F-G \in \widetilde{\mathcal{I}}$, that is

$$
\{f, \phi(g)\}+\{\phi(f), g\}-\phi(\{f, g\}) \in \mathcal{I}
$$

This means exactly that $\phi \in \operatorname{Ker}\left(\delta^{1}\right)$.

In order to interpret the second cohomology group, we define the bilinear mapping

$$
\begin{aligned}
\widetilde{o b}: \mathcal{C}_{L}^{1} \times \mathcal{C}_{L}^{1} & \longrightarrow \mathcal{C}_{L}^{2} \\
(\Phi, \Psi) & \longmapsto(g \wedge h \mapsto\{\Phi(g), \Psi(h)\})
\end{aligned}
$$

In this way we get a quadratic form $o b(\Phi):=\widetilde{o b}(\Phi, \Phi)$. It can be immediately verified that this induces a map $o b: \mathcal{H}^{1}\left(\mathcal{C}_{L}^{\bullet}\right) \rightarrow \mathcal{H}^{2}\left(\mathcal{C}_{L}^{\bullet}\right)$. We will now prove the following: Given a lagrangian deformation $\Phi \in \mathcal{L} \mathcal{T}_{L}^{1}$. Then there is a lift to second order defining an involutive ideal iff $o b(\Phi)=0 \in \mathcal{L} \mathcal{T}_{L}^{2}$. The last condition is equivalent to the existence of $\Psi \in \mathcal{L T}_{L}^{1}$ with $o b(\Phi)=\delta(\Psi)$, i.e.

$$
\{\Phi(f), \Phi(g)\}=\Psi(\{f, g\})-\{f, \Psi(g)\}-\{\Psi(f), g\} \quad \forall f, g \in \mathcal{L}
$$

But this means that the following ideal is involutive.

$$
J=\left(f_{1}+\epsilon \Phi\left(f_{1}\right)+\epsilon^{2} \Psi\left(f_{1}\right), \ldots, f_{k}+\epsilon \Phi\left(f_{k}\right)+\epsilon^{2} \Psi\left(f_{k}\right)\right)
$$

Remark: The fact that $\mathcal{L T}_{L}^{2}$ is not the real obstruction space make precise what was meant by saying that complex $\mathcal{C}_{L}^{\bullet}$ is a first approximation of the object we are looking for: Hopefully, there is a modified version of this complex whose cohomology gives, in complete analogy with the cotangent complex, the spaces $T^{1}$ and $T^{2}$ for flat lagrangian deformations. On the other hand, it is perhaps not even necessary to impose flatness as the involutivity condition implies that the dimension cannot drop, see also Mat.

Corollary 2. There is an exact sequence

$$
0 \rightarrow H^{1}\left(L, \mathbb{K}_{L}\right) \rightarrow \mathbb{H}^{1}\left(\mathcal{C}_{L}^{\bullet}\right) \rightarrow H^{0}\left(L, \mathcal{L T}_{L}^{1}\right) \rightarrow H^{2}\left(L, \mathbb{K}_{L}\right) \rightarrow \mathbb{H}^{1}\left(\mathcal{C}_{L}^{\bullet}\right)
$$

Furthermore, there are two special cases:

- Let $L$ be a contractible space. Then $\mathbb{H}^{1}\left(\mathcal{C}_{L}^{\bullet}\right)=H^{0}\left(L, \mathcal{L} \mathcal{T}_{L}^{1}\right)$ and in fact: $L T_{L}^{1}=H^{0}\left(L, \mathcal{L} T_{L}^{1}\right)$.

- Let $L$ be Stein and smooth. Then it follows that $\mathbb{H}^{1}\left(\mathcal{C}_{L}^{\bullet}\right)=H^{1}\left(L, \mathbb{K}_{L}\right)$ and the space of global deformations is indeed $L T_{L}^{1}=H^{1}\left(L, \mathbb{K}_{L}\right)$. 
Proof. The first fact is just the definition of the sheaf $\mathcal{L T}_{L}^{1}$. In the second case, note that the space of embedded flat deformations is $H^{0}\left(L, \mathcal{N}_{L}\right)$, where $\mathcal{N}_{L}$ is the normal bundle of $L$ in $M$. As $L$ is smooth, this happens to be $H^{0}\left(L, \Omega_{L}^{1}\right)$, so each infinitesimal flat deformation corresponds to globally defined one-form on $L$. It is closed iff the deformation is lagrangian and the subspace of exact one-forms are deformations induced by hamiltonian vector fields (isodrastic deformations, see [Wei90]), these are the trivial ones. $L$ is assumed to be a Stein manifold, in this case the first de Rham-cohomology group is exactly $H^{1}\left(L, \mathbb{K}_{L}\right)$.

By analogy with the cotangent complex, the following generalization is probably true although we did not check the details.

Proposition 1. The space of infinitesimal lagrangian deformations of a complex space $L$ which is a lagrangian subvariety of a symplectic manifold $(M, \omega)$ is given by

$$
L T_{L}^{1}=\mathbb{H}^{1}\left(\mathcal{C}_{L}^{\bullet}\right)
$$

\section{Finiteness of the cohomology}

This section is devoted to the proof of the following result.

Theorem 2. Let $L \subset M$ be a lagrangian subvariety as above. Assume that the following condition is satisfied: Denote by edim( $p)$ the embedding dimension of a point $p \in L$, that is edim $(p):=\operatorname{dim}_{\mathbb{K}}\left(\mathbf{m}_{p} / \mathbf{m}_{p}^{2}\right)$, where $\mathbf{m}_{p}$ is the maximal ideal in the local ring $\mathcal{O}_{(L, p)}$. Let $S_{k}^{L}$ be the following set

$$
S_{k}^{L}:=\{p \in L \mid \operatorname{edim}(p)=2 n-k\} \subset L
$$

for all $k \in\{0, \ldots, n\}$, then suppose that we have

$$
\operatorname{dim}\left(S_{k}^{L}\right) \leq k
$$

for all $k$. Under this condition (which will be called "condition P"), all $\mathcal{H}^{i}\left(\mathcal{C}_{L}^{\bullet}\right)$ are constructible sheaves of $\mathbb{K}$-vector spaces with respect to the stratification given by the $S_{k}^{L}$.

Before going into the details of the proof, we would like to explain the meaning of the condition (2).

Lemma 4. Let $p \in S_{k}^{L} \subset L$ with $k>0$. Then the germ $(L, p)$ can be decomposed into a product

$$
(L, p)=\left(L^{\prime}, p^{\prime}\right) \times(\mathbb{K}, 0)
$$

where $\left(L^{\prime}, p^{\prime}\right)$ is a germ of a lagrangian variety in the symplectic space $\mathbb{K}^{2 n-2}$. Furthermore, we have $p^{\prime} \in S_{k-1}^{L^{\prime}}$.

Proof. Let $x_{1}, \ldots, x_{2 n}$ be coordinates of $M$ centered at $p$. Then the fact that $\operatorname{edim}(p)<2 n$ implies that there are coefficients $\alpha_{i} \in \mathcal{O}_{L, p}$ such that the following equation holds in $\mathcal{O}_{L, p}$

$$
\sum_{i=1}^{2 n} \alpha_{i} x_{i}+h=0
$$


where $h$ is an element of $\mathcal{O}_{L, p}$ vanishing at second order. So we have an element in the ideal describing $(L, p)$ whose derivative do not vanish. Then $(L, p)$ is fibred by the hamiltonian flow of this function. Explicitly, we can make an analytic change of coordinates, such that $\alpha_{1}=1, \alpha_{i}=0$ for all $i>1$ and $h=0$. Than the ideal of $(L, p)$ is of the form $\left(x_{1}, f_{1}, \ldots, f_{m}\right)$ for some functions $f_{i}$ which are independent of the variable $x_{n+1}$ (provided that we have chosen the symplectic form to be $\left.\sum_{i=1}^{n} d x_{i} \wedge d x_{n+i}\right)$.

According to the lemma, the set of points of the variety $L$ can be divided into two classes, those with maximal embedding dimension (these are the "bad points") and those (with $\operatorname{edim}(p)<2 n$ ) at which $L$ is decomposable. Condition P implies that the bad points are isolated. As usual, the proof of the theorem consists of two parts: First, we will show that the cohomology sheaves are locally constant on the strata $S_{k}^{L}$. This is an immediate consequence of the following lemma. Then it suffices to show that all stalks of $\mathcal{H}^{p}\left(\mathcal{C}_{L}^{\bullet}\right)$ are finite-dimensional.

Lemma 5 (Propagation of Deformations). Let

$$
(L, 0) \subset\left(\mathbb{K}^{2 n}, 0\right)
$$

be a germ of a lagrangian subvariety which can be decomposed, i.e., there is a germ $\left(L^{\prime}, 0\right)$ (which is lagrangian in $\left(\mathbb{K}^{2 n-2}, 0\right)$ ) such that $(L, 0)=\left(L^{\prime}, 0\right) \times$ $(\mathbb{K}, 0)$. Denote by $\pi: L \rightarrow L^{\prime}$ the projection. Then there is a quasi-isomorphism of sheaf complexes

$$
j: \pi^{-1} \mathcal{C}_{L^{\prime}}^{\bullet} \rightarrow \mathcal{C}_{L}^{\bullet}
$$

Proof. The proof of lemma 1 shows that the ideals $I$ and $I^{\prime}$ describing the two germs differ by exactly one element whose differential do not vanish at the origin. This implies that the conormal sheaves $\mathcal{L}$ of $L$ and $\mathcal{L}^{\prime}$ of $L^{\prime}$ are related by the formula $\mathcal{L}=\pi^{*} \mathcal{L}^{\prime} \oplus \mathcal{O}_{L}$. It follows that

$$
\mathcal{C}_{L}^{p}=\operatorname{Hom}_{\mathcal{O}_{L}}\left(\pi^{*} \bigwedge^{p} \mathcal{L}^{\prime}, \mathcal{O}_{L}\right) \oplus \mathcal{H o m}_{\mathcal{O}_{L}}\left(\pi^{*} \bigwedge^{p-1} \mathcal{L}^{\prime}, \mathcal{O}_{L}\right)
$$

Now we have to describe the differential on $\mathcal{C}_{L}^{\bullet}$. We choose local Darboux coordinates $\left(p_{1}, \ldots, p_{n}, q_{1}, \ldots, q_{n}\right)$ on $\mathbb{K}^{2 n}$ and $\left(p_{2}, \ldots, p_{n}, q_{2}, \ldots, q_{n}\right)$ on $\mathbb{K}^{2 n-2}$. Suppose that the two ideals are $I=\left(f_{1}, \ldots, f_{m}, p_{1}\right)$ and $I^{\prime}=\left(f_{1}, \ldots, f_{m}, p_{1}, q_{1}\right)$ (if we consider $L^{\prime}$ as embedded in $\mathbb{K}^{2 n}$ ). Let $\Phi$ be an element of

$$
\operatorname{Hom}_{\mathcal{O}_{L}}\left(\pi^{*} \bigwedge^{p} \mathcal{L}^{\prime}, \mathcal{O}_{L}\right)
$$

Then it can be written as a power series in $q_{1}$ with coefficients in $\mathcal{C}_{L^{\prime}}^{\bullet}$. A direct calculation shows that the differential on $\mathcal{C}_{L}^{\bullet}$ is

$$
\begin{aligned}
& \delta: \quad \mathcal{C}_{L}^{p} \quad \longrightarrow \quad \mathcal{C}_{L}^{p+1} \\
& \sum_{i=0}^{\infty}(\Phi, \Psi) q_{1}^{i} \quad \mapsto \quad \sum_{i=0}^{\infty}\left(\delta \Phi_{i}, \delta \Psi_{i}+(-1)^{p+1}(i+1) \Phi_{i+1}\right) q_{1}^{i}
\end{aligned}
$$

It is clear that the morphism $j$ must be the obvious inclusion

$$
\mathcal{H}_{m_{\mathcal{O}^{\prime}}}\left(\bigwedge^{p} \mathcal{L}^{\prime}, \mathcal{O}_{L^{\prime}}\right) \hookrightarrow \mathcal{H} m_{\mathcal{O}_{L}}\left(\pi^{*} \bigwedge^{p} \mathcal{L}^{\prime}, \mathcal{O}_{L}\right) \oplus \mathcal{H o m}_{\mathcal{O}_{L}}\left(\pi^{*} \bigwedge^{p-1} \mathcal{L}^{\prime}, \mathcal{O}_{L}\right)
$$


We will now show that the cokernel of this inclusion is acyclic. Then it follows immediately that $j$ induces an isomorphism on the cohomology. So let $\Gamma$ be an element of $\operatorname{Coker}(j) \cap \operatorname{Ker}(\delta)$, that is,

$$
\Gamma=\sum_{i=1}^{\infty}\left(\Phi_{i}, \Psi_{i}\right) q_{1}^{i}+\left(0, \Psi_{0}\right)
$$

where $\delta \Phi_{i}=0$ and $\delta \Psi_{i}=(-1)^{p}(i+1) \Phi_{i+1}$ for all $i$. But then $\Gamma$ vanishes in the cohomology because it can be written as $\Gamma=\delta \Lambda$ with

$$
\Lambda:=\sum_{i=1}^{\infty}\left(\frac{(-1)^{p} \Psi_{i-1}}{i}, 0\right) q_{1}^{i} \in \mathcal{C}_{L}^{p-1}
$$

Corollary 3. We have isomorphisms of sheaves

$$
\pi^{-1} \mathcal{H}^{i}\left(\mathcal{C}_{L^{\prime}}^{\bullet}\right) \cong \mathcal{H}^{i}\left(\mathcal{C}_{L}^{\bullet}\right)
$$

Proof. This is obvious since $\pi^{-1}$ is an exact functor.

Let $p \in S_{k}^{L}$ be a point at which $L$ is decomposable, i.e. $k>0$. By induction, we find a neighborhood $U \subset L$ of $p$ such there is an analytic isomorphism $h$ : $U \stackrel{\cong}{\longrightarrow} Z \times B_{\epsilon}(0)^{k}$, where $Z$ is lagrangian in $\mathbb{K}^{2(n-k)}, B_{\epsilon}(0):=\{z \in \mathbb{K}|| z \mid<\epsilon\}$ and each $q \in U \cap S_{l}^{L}$ corresponds via $h$ to a point $\left(q^{\prime}, b\right) \in Z \times B(\epsilon)^{k}$ with $q^{\prime} \in S_{l-k}^{Z}$. In particular, the image of $U \cap S_{k}^{L}$ under $h$ is $\left(\{p t\}, B(\epsilon)^{k}\right)$, so by the last corollary, $\mathcal{H}^{p}\left(\mathcal{C}_{L}^{\bullet}\right)$ is constant on $U \cap S_{k}^{L}$.

It remains to show that the stalks of the cohomology are finite-dimensional. Again by corollary 3, this is done once we have shown it for points with maximal embedding dimension. We will use a method developed in [BG80]. In this paper, the following situation is considered. Let $f: X \rightarrow S$ be a morphism of complex spaces (with $\operatorname{dim}(S)=1$ ) and $\mathcal{K}^{\bullet}$ a certain sheaf complex on $X$. Then, under suitable conditions, the relative hypercohomology $\mathbb{R}^{i} f_{*} \mathcal{K} \bullet$ are coherent sheaves of $\mathcal{O}_{S}$-modules. The proof of this theorems relies on a functional analytic argument of Kiehl and Verdier (see [KV71] or [Dou74]) which states, roughly speaking, that if the mapping induced on the complex of sections of $\mathcal{K}^{\bullet}$ by a small shrinking of the open set (over which the sections are taken) is a quasi-isomorphism, then the hypercohomology groups are finite dimensional vector spaces. We are going to use this result in the form of [vS87].

Lemma 6. Let $(L, p) \in\left(\mathbb{K}^{2 n}, 0\right)$ be a germ of a lagrangian variety satisfying condition $P$ which is indecomposable at $p$. Then the stalk $\mathcal{H}^{i}\left(\mathcal{C}_{L}\right)_{p}$ is a finitedimensional $\mathbb{K}$-vector space.

Proof. Choose a representative $V$ for the the germ such that $\operatorname{edim}(q)=2 n$ iff $q=p$ for all points $q \in V$. We refer the reader to theorem 1 in vS87. We do not consider a relative situation here, so the map $f: X \rightarrow S$ in this theorem is replaced by $V \rightarrow\{0\}$ (Obviously, $V$ can be chosen such that this map is a standard representative of the germ $(L, p)$ in the sense of definition 1 in |vS87], i.e., $\left.V=L \cap B_{\epsilon}(p)\right)$. The complex of sheaves in the theorem is the complex $\mathcal{C}_{L}^{\bullet}$, which satisfies the first two properties $\left(C_{L}^{p}\right.$ is $\mathcal{O}_{L}$-coherent and the differential is 
$\mathbb{K}$-linear). Our task is to verify the third axiom, that is, we have to find a vector field of class $C^{\infty}$ such that $\mathcal{H}^{p}\left(\mathcal{C}_{L}^{\bullet}\right)$ is transversally constant (see definition 2 in [VS87]), this will be done in corollary 4. Now the proof of the theorem shows that there is a smaller neighborhood $V_{1}$ of $p$ such that $\Gamma\left(V, \mathcal{H}^{p}\left(\mathcal{C}_{L}^{p}\right)\right)=\Gamma\left(V_{1}, \mathcal{H}^{p}\left(\mathcal{C}_{L}^{p}\right)\right)$. This gives the result by using [KV71] in the same way as in [vS87] or [BG80].

Lemma 7. Let $q \in V \cap S_{k}^{L}$ with $k>0$. Then there is a $C^{\infty}$-vectorfield in a neighborhood $W$ of $q$ in $M$, tangent to $V \cap S_{k}^{L}$ and transversal to $\partial B_{\epsilon}(p)$.

Proof. It follows from lemma 1 that there exist $k$ linear independent hamiltonian vector fields on $M$ which respects the stratum $S_{L}^{k}$. Now we have to distinguish the cases $\mathbb{K}=\mathbb{R}$ and $\mathbb{K}=\mathbb{C}$, in the first one, since $S_{L}^{k}$ is of real dimension $k$ and since the intersection of $L$ and $B_{\epsilon}(p)$ was transversal, it follows immediately that we can find a linear combination of theses $C^{\infty}$-fields which is transversal to $B_{\epsilon}(p)$. The same is true in the complex case, here we have $k$ independent hamiltonian fields $\eta_{1}, \ldots, \eta_{k}$ which are holomorphic. As the holomorphic tangent space at each point is canonically isomorphic (over $\mathbb{R}$ ) to the real one, we get $2 k$ linear independent $C^{\infty}$-fields by applying this isomorphism to $\eta_{1}, \ldots, \eta_{k}, i \eta_{1}, \ldots, i \eta_{k}$. These can be used to find a field transversal to $\partial B_{\epsilon}(p)$.

Corollary 4. There is a $C^{\infty}$-vectorfield $\vartheta$ on a neighborhood $U$ of $\partial B_{\epsilon}(p)$ in $M$ such that $\mathcal{H}^{p}\left(\mathcal{C}_{L}^{\infty}\right)$ is transversally constant with respect to $U$ and $\vartheta$.

Proof. Set $U:=(V \backslash\{p\})^{\circ}$. Then the last lemma yields a covering $U_{i}$ of $U$ and vector fields $\vartheta_{i}$ defined in a neighborhood of $U_{i}$ in $M$. Chose a partition of unity subordinate to this covering to obtain a field on $U$ which is still transversal to $\partial B_{\epsilon}$. For each point $q \in U$, which is contained in some stratum $S_{k}^{L}, \vartheta$ is necessarily tangent to $S_{k}^{L}$, so the cohomology sheaves are constant on the local integral curves of $\vartheta$.

Remark: By the Riemann-Hilbert-correspondence (see Bjö93]), the complex $\mathcal{H}^{\bullet}:=\mathcal{H}\left(\mathcal{C}_{L}^{\bullet}\right)$, viewed as an object of $\mathcal{D}_{c}^{b}\left(\mathbb{K}_{M}\right)$ (the derived category of constructible sheaves of $\mathbb{K}$-vector spaces on $M$ ) corresponds via the de Rhamfunctor to a unique complex of coherent $\mathcal{D}_{M}$-modules with regular holonomic cohomology supported on $L$ (i.e., an object of $\mathcal{D}_{\text {r.h. }}^{b}\left(\mu_{L}\left(\mathcal{D}_{M}\right)\right)$ ).

Lemma 8. The complex $\mathcal{H}^{\bullet}$ satisfies the first perversity condition, that is, the following inequality holds.

$$
\operatorname{dim}\left(\mathcal{H}^{i}\left(\mathcal{C}_{L}^{\bullet}\right)\right) \leq n-i
$$

Proof. Let $p \in S_{k}^{L}$. Then $(L, p)=\left(L^{\prime}, p^{\prime}\right) \times\left(\mathbb{K}^{k}, 0\right)$ and $\mathcal{H}^{i}\left(\mathcal{C}_{L}^{\bullet}\right)_{p}=\mathcal{H}^{i}\left(\mathcal{C}_{L^{\prime}}^{\bullet}\right)_{p^{\prime}}$. But $\operatorname{dim}\left(L^{\prime}\right) \leq n-k$, so $\mathcal{H}^{i}\left(\mathcal{C}_{L^{\prime}}\right)_{p^{\prime}}=0$ for all $i>n-k$.

In case that the second perversity condition is also satisfied, the $\mathcal{H}^{i}$ 's are the de Rham-cohomology modules of some $\mathcal{D}_{M}$-module supported on $L$. The following consideration gives more evidence that the complex $\mathcal{C}_{L}^{\bullet}$ is closely related to $\mathcal{D}$-module theory: Every complex manifold is lagrangian in its own cotangent bundle. Consider Spencer's complex, which is a resolution of $\mathcal{O}_{X}$ as a $\mathcal{D}_{X}$-module, explicitly:

$$
S p\left(\mathcal{O}_{X}\right)^{\bullet}: \quad \ldots \rightarrow \mathcal{D}_{X} \otimes_{\mathcal{O}_{X}} \Theta_{X}^{p+1} \rightarrow \mathcal{D}_{X} \otimes_{\mathcal{O}_{X}} \Theta_{X}^{p} \rightarrow \ldots \mathcal{D}_{X} \rightarrow \mathcal{O}_{X} \rightarrow 0
$$


The de Rham-complex of $\mathcal{D}_{X}$-module $\mathcal{M}$ is obtained as

$$
D R(M):=\operatorname{Hom}_{\mathcal{D}_{X}}\left(\operatorname{Sp}\left(\mathcal{O}_{X}\right)^{\bullet}, \mathcal{M}\right)
$$

If we define a generalized version of the complex $\mathcal{C}_{L}^{\bullet}$ as

$$
\mathcal{C}_{L}^{p}(\mathcal{M}):=\mathcal{H} m_{\mathcal{O}_{L}}\left(\bigwedge^{p} \mathcal{L}, \mathcal{M}\right)
$$

for some module $\mathcal{M}$ over the Lie algebroid $\mathcal{L}$, then $\mathcal{C}_{X}^{p}(\mathcal{M})$ (for $X$ lagrangian in $\left.T^{*} X\right)$ is exactly the de Rham-complex of the $\mathcal{D}_{X}$-Module $\mathcal{M}$.

\section{$5 \quad$ Examples and results}

In this section we will describe some of the basic examples of singular lagrangian submanifolds, in particular those for which results on their deformation spaces are available. We start with the easiest case, a plane curve $C$ in $\mathbb{K}^{2}$, given as the zero set of a mapping $f: \mathbb{K}^{2} \rightarrow \mathbb{K}$. Such a curve $C$ is obviously lagrangian. In this case the complex $\mathcal{C}_{C}^{\bullet}$ is simplifies to

$$
\begin{aligned}
\mathcal{C}_{C}^{0}=\mathcal{O}_{C} & \stackrel{\delta}{\longrightarrow} \mathcal{C}_{C}^{1}=\mathcal{H o m}_{\mathcal{O}_{C}}\left(\mathcal{I} / \mathcal{I}^{2}, \mathcal{O}_{C}\right)=\text {Hom}_{\mathcal{O}_{C}}\left(\mathcal{O}_{C}, \mathcal{O}_{C}\right)=\mathcal{O}_{C} \\
h & \longmapsto\{h, f\}
\end{aligned}
$$

It follows immediately that $\mathcal{H}^{2}\left(\mathcal{C}_{C}^{\bullet}\right)=0$, while $\mathcal{L T}_{C}^{1}=\mathcal{H}^{1}\left(\mathcal{C}_{C}^{\bullet}\right)=\operatorname{Coker}(\delta)$. This sheaf is supported on the singular points of the curve, let $x_{0}$ be such a point. Then we have

$$
\mathcal{L} \mathcal{T}_{C, x_{0}}^{1}=\frac{\mathcal{O}_{C, x_{0}}}{\left\{\{h, f\} \mid h \in \mathcal{O}_{C, x_{0}}\right\}}
$$

Now the following equalities hold

$$
\begin{aligned}
\frac{\mathcal{O}_{C, x_{0}}}{\left\{\{h, f\} \mid h \in \mathcal{O}_{C, x_{0}}\right\}} & =\frac{\Omega_{\mathbb{K}^{2}, x_{0}}^{2}}{\left\{f \Omega_{\mathbb{K}^{2}, x_{0}}^{2}+\left\{d f \wedge d h \mid h \in \mathcal{O}_{C, x_{0}}\right\}\right\}} \\
& =\frac{\Omega_{\mathbb{K}^{2}, x_{0}}^{2}}{\left\{f \Omega_{\mathbb{K}^{2}, x_{0}}^{2}+d f \wedge d \Omega_{\mathbb{K}^{2}, x_{0}}^{0}\right\}}
\end{aligned}
$$

because $\mathcal{O}_{C, x_{0}} \cong \Omega_{\mathbb{K}^{2}, x_{0}}^{2} /\left(f \Omega_{\mathbb{K}^{2}, x_{0}}^{2}\right)$ and the Poisson bracket of two functions $f$ and $g$ corresponds under the isomorphism $\mathcal{O}_{\mathbb{K}^{2}, x_{0}} \cong \Omega_{\mathbb{K}^{2}, x_{0}}$ to the 2-form $d f \wedge d g$. But it is known (see Mal74) that the dimension of the last quotient equals $\mu$, the Milnor number of the plane curve singularity $\left(C, x_{0}\right)$. So the result is:

$$
\mathcal{L} \mathcal{T}_{C}^{1}=\prod_{x_{0} \in \operatorname{Sing}(C)} \mathbb{K}^{\mu\left(C, x_{0}\right)}
$$

This is remarkable because the usual $T_{C}^{1}$ has dimension $\tau$ (the Tjurina number) which is in general smaller than $\mu$. The difference corresponds to the space of deformations of the restriction of the symplectic structure to $L$ (see also [Giv88]).

Applying lemma 5 , we see that the dimension of $\mathcal{L T}^{1}$ for a surface singularity which is a curve germ, crossed with a smooth factor is also equal to the Milnor 
number of this curve. This result can also be obtained by a direct calculus, e.g., for a cuspidal edge given in four-space (with coordinates $A, B, C, D$ and symplectic form $d A \wedge d C+d B \wedge d D$ ) by the two equations $A, B^{2}-C^{3}$, we get $L T^{1}=\mathbb{K}^{2}$ and $L T^{2}=0$.

We will proceed with further examples of lagrangian surfaces in $\mathbb{K}^{4}$, which satisfy condition $\mathrm{P}$ of theorem 2. So there are three strata: one point with embedding dimension four (supposed to be the origin), the singular locus away from this point and the regular locus. In order to simplify the calculation of the cohomology of $\mathcal{C}^{\bullet}$, we will suppose that our varieties are strongly quasihomogeneous in the sense of [CJNMM96], that is, one can choose local coordinates of the ambient space around each point of $L$ such that the defining equations become weighted homogeneous with positive weights. In this case, the de Rham-complex is a resolution of the constant sheaf as one can see by considering the decomposition of the modules $\Omega_{L}^{p}$ into eigenspaces of the Liederivative.

Lemma 9. Let $L \subset M$ be a strongly quasi-homogeneous lagrangian subvariety. Consider the map $J:\left(\Omega_{L}^{\bullet}, d, \wedge\right) \rightarrow\left(\mathcal{C}_{L}^{\bullet}, \delta, \wedge\right)$ of DGA's from corollary $\mathbb{\text { A. Denote }}$ by $\widetilde{\Omega}_{L}^{\bullet}$ the subcomplex $\operatorname{Im}(J)$ in $\mathcal{C}_{L}^{\bullet}$. Then $\widetilde{\Omega}_{L}^{\bullet}$ is a resolution of $\mathbb{K}_{L}$.

Proof. By the long exact cohomology sequence, it suffices to prove that the complex $\operatorname{Ker}(J)$ is acyclic. This can be done in exactly the same way as for $\Omega_{L}^{\bullet}$ provided that the inner derivative $i_{E}$ ( $E$ being the quasi-homogeneous Euler vector field) maps $\mathcal{K} \operatorname{er}(J) \cap \Omega_{L}^{p}$ into $\mathcal{K} \operatorname{er}(J) \cap \Omega_{L}^{p-1}$. But this follows from lemma 1 because if $\omega$ is a torsion element than the same holds for $i_{E} \omega$.

Corollary 5. Denote by $\mathcal{G}_{L}^{\bullet}$ the cokernel of the map J. Then there is an exact sequence of $\mathcal{O}_{L}$-modules

$$
0 \longrightarrow \widetilde{\Omega}_{L}^{\bullet} \longrightarrow \mathcal{C}_{L}^{\bullet} \longrightarrow \mathcal{G}_{L}^{\bullet} \longrightarrow 0
$$

and the long associated long exact sequence gives

$$
\mathcal{H}^{i}\left(\mathcal{C}_{L}^{\bullet}\right)=\mathcal{H}^{i}\left(\mathcal{G}_{L}^{\bullet}\right)
$$

for all $i \geq 0$. In particular, if $L$ is of dimension two, then we get

$$
\begin{aligned}
\mathcal{H}^{1}\left(\mathcal{C}_{L}^{\bullet}\right) & =\operatorname{Ker}\left(\delta: \mathcal{G}_{L}^{1} \rightarrow \mathcal{G}_{L}^{2}\right) \\
\mathcal{H}^{2}\left(\mathcal{C}_{L}^{\bullet}\right) & =\operatorname{Coker}\left(\delta: \mathcal{G}_{L}^{1} \rightarrow \mathcal{G}_{L}^{2}\right)
\end{aligned}
$$

We can thus calculate $\mathcal{L T}_{L}^{1}$ and $\mathcal{L T}_{L}^{2}$ by computing the induced morphism $\delta: \mathcal{G}_{L}^{1} \rightarrow \mathcal{G}_{L}^{2}$. As $J$ is an isomorphism at smooth points, the sheaves $\mathcal{G}_{L}^{i}$ are supported on the singular locus of $L$, which is of dimension one. In a neighborhood of all of its regular points $q$ (points with embedding dimension three), the germ is decomposable and the dimension of $\mathcal{H}^{i}\left(\mathcal{C}_{L}^{\bullet}\right)_{q}$ is given by lemma 5 . So we are only interested in the one special point with maximal embedding dimension. We now choose an element $p \in \mathcal{O}_{L}$ which is finite when restricted to the support of $\mathcal{G}_{L}^{i}$, note that although this is set-theoretically equal to the singular locus of $L$, it may have embedded components. We will suppose that $p$ maps the origin in $\mathbb{K}^{4}$ to the origin in $\mathbb{K}$. Consider the sheaves $p_{*} \mathcal{G}_{L}^{1}$ and $p_{*} \mathcal{G}_{L}^{2}$, these are modules over $\mathcal{O}_{\mathbb{K}}$. Denote by $\widetilde{E}$ resp. $\widetilde{F}$ the modules of section of $p_{*} \mathcal{G}_{L}^{1}$ resp. $p_{*} \mathcal{G}_{L}^{2}$ in 
a small neighborhood of the origin. Then they can be decomposed into torsion and torsion free parts, the former being supported on the origin while the latter is free over $\mathbb{K}\{t\}$. In practice, this is done as follows: As $\mathcal{G}_{L}^{1}$ and $\mathcal{G}_{L}^{2}$ are graded modules over $\mathcal{O}_{L}$ and the map $\delta: \mathcal{G}_{L}^{1} \rightarrow \mathcal{G}_{L}^{2}$ is homogeneous, we consider the decomposition of these modules into homogeneous parts. The map $p$ is finite, so the torsion submodules of $\widetilde{E}$ and $\widetilde{F}$ corresponds to homogeneous parts of $\mathcal{G}_{L}^{1}$ and $\mathcal{G}_{L}^{2}$ in a finite number of degrees. This yields a decomposition of $\widetilde{E}$ and $\widetilde{F}$ into $\widetilde{E}=\widehat{E} \oplus E$ and $\widetilde{F}=\widehat{F} \oplus F$ such that $\widehat{E}$ and $\widehat{F}$ are supported on the origin, while $E$ and $F$ are free. $\widehat{E}$ and $\widehat{F}$ being artinian, the kernel and cokernel of $\delta_{\mid \widehat{E}}$ can be computed explicitly. The following lemma is used to do this for $\delta_{\mid E}$.

Lemma 10. The rank of $E$ and $F$ is the Milnor number $\mu$ of the transversal curve singularity, i.e. the germ $\left(L^{\prime}, 0\right)$ such that $(L, p)=\left(L^{\prime}, 0\right) \times(\mathbb{K}, 0)$ for all $p \in \operatorname{Sing}(L) \backslash 0$. Therefore, $\delta_{\mid E}: E \rightarrow F$ is an $(E, F)$-connection in the sense of Mal74.

Proof. This is an explicit calculation involving the definition of the complex $\mathcal{C}_{L}^{\bullet}$ and the map $J: \Omega_{L}^{\bullet} \rightarrow \mathcal{C}_{L}^{\bullet}$. It suffices to calculate the rank of $\left(\mathcal{G}_{L}^{1}\right)_{p}$ and $\left(\mathcal{G}_{L}^{2}\right)_{p}$. So suppose that $(L, p)$ is a decomposable germ. We choose coordinates $(x, y, s, t) \in \mathbb{K}^{4}$ (with symplectic form $\omega=d x \wedge d y+d s \wedge d t$ ) around $p$ such that $L$ is given as the zero locus of $s$ and a function $f$ depending only on $x$ and $y$. Denote the ideal generated by these two functions by $I$ and by $R$ the stalk of $\mathcal{O}_{L}$ at the point $p$. Then we can identify $I / I^{2}$ with $R^{2}$, so $\operatorname{Hom}_{R}\left(I / I^{2}, R\right)$ is free on the two generators $n_{1}$ and $n_{2}$, where

$$
\begin{array}{ll}
n_{1}(f)=1 & n_{1}(s)=0 \\
n_{2}(f)=0 & n_{2}(s)=1
\end{array}
$$

while $\operatorname{Hom}_{R}\left(I / I^{2} \wedge I / I^{2}, R\right)$ is just $R$, generated by the homomorphism sending $f \wedge s$ to 1 in $R$. The complex $\mathcal{C}^{\bullet}$ at the point $p$ then reads:

$$
\begin{aligned}
& R \quad \longrightarrow \quad R n_{1} \oplus R n_{2} \quad \longrightarrow \quad R \\
& h \longmapsto(\{h, f\},\{h, s\}) \\
& (p, q) \quad \longmapsto\{p, s\}+\{f, q\}
\end{aligned}
$$

where the pair $(p, q) \in R^{2}=\operatorname{Hom}_{R}\left(I / I^{2}, R\right)$ denotes the homomorphism sending $f \in I / I^{2}$ to $p \in R$ and $s \in I / I^{2}$ to $q \in R$.

Now we have to investigate the modules of differential forms on $L$ at $x$. In general

$$
\Omega_{R}^{p}=\Omega_{S}^{p} /\left(I \Omega_{S}^{p}+d I \wedge \Omega_{S}^{p-1}\right)
$$

where $S$ is the ring $\mathbb{K}\{x, y, s, t\}$. This leads to

$$
\begin{aligned}
& \Omega_{R}^{1}=M_{1} \oplus M_{2} \\
& \Omega_{R}^{2}=M_{3} \oplus M_{4}
\end{aligned}
$$

where we have used the following abbreviations:

$$
\begin{aligned}
& M_{1}=\frac{R d x \oplus R d y}{R d f} \\
& M_{2}=R d t \\
& M_{3}=\frac{R d x \wedge d y}{R d f \wedge d x \oplus R d f \wedge d y} \\
& M_{4}=\frac{R d x \wedge d t \oplus R d y \wedge d t}{R d f \wedge d t}
\end{aligned}
$$


$J: \Omega_{L}^{\bullet} \rightarrow \mathcal{C}_{L}^{\bullet}$ can be described as

$$
\begin{aligned}
& J: M_{1} \quad \longrightarrow \quad R n_{1} \oplus R n_{2} \\
& d x \longmapsto(\{x, f\},\{x, s\})=\left(\partial_{y} f, 0\right) \\
& d y \longmapsto(\{y, f\},\{y, s\})=\left(-\partial_{x} f, 0\right) \\
& J: M_{2} \quad \longrightarrow \quad R n_{1} \oplus R n_{2} \\
& d t \longmapsto(\{t, f\},\{t, s\})=(0,1) \\
& J: M_{3} \longrightarrow R \\
& d x \wedge d y \quad \longmapsto \quad J(d x) \wedge J(d y)=0 \\
& J: M_{4} \longrightarrow R \\
& d x \wedge d t \longmapsto J(d x) \wedge J(d t)=\partial_{y} f \\
& d y \wedge d t \longmapsto J(d x) \wedge J(d t)=-\partial_{x} f
\end{aligned}
$$

$E$ and $F$ are the cokernels of the maps $J: M_{1} \oplus M_{2} \rightarrow R n_{1} \oplus R n_{2}$ and $J: M_{3} \oplus M_{4} \rightarrow R$, respectively. So the result is

$$
E=F=R /\left(\partial_{x} f, \partial_{y} f\right)=\mathbb{K}\{t\} \otimes \mathcal{O}_{L^{\prime}, p} /\left(\partial_{x} f, \partial_{y} f\right)=\mathbb{K}\{t\}^{\tau}
$$

As $L$ is strongly quasi-homogeneous, we have weighted homogeneous local equations for the transversal slice which gives $\tau=\mu$.

Denote $\delta_{\mid E}$ by $D$ for short. Then $D$ is a first-order differential operator $D$ : $\mathcal{O}_{\mathbb{K}}^{\mu} \rightarrow \mathcal{O}_{\mathbb{K}}^{\mu}$ which respects the grading. So it is of the form

$$
D=t \partial_{t} \mathbf{1}+A
$$

where $A$ is a constant $\mu \times \mu$-matrix. Thus, the second part of the cohomology of $\mathcal{C}_{L}^{\bullet}$ (i.e. kernel and cokernel of $\delta$ ) can be deduced from the solutions of the differential system given by $D$. All explicit calculations have been done using Macaulay2.

The first interesting example we are going to study is the so called "open swallowtail". For details of its definition, see Giv82 and Giv83. Consider the space of polynomials in one variable of degree $d:=2 k+1$ with fixed leading coefficient and sum of roots equal to zero, that is, the space

$$
\mathcal{P}_{2 k+1}=\left\{x^{2 k+1}+A_{2} x^{2 k-1}+\ldots+A_{2 k+1} x^{0}\right\} \cong \mathbb{K}^{2 k}
$$

which comes equipped with the following symplectic structure

$$
\omega=\sum_{i=2}^{k+1}(2 k+1-i) !(i-2) ! \cdot(-1)^{i} d A_{i} \wedge d A_{2 k+3-i}
$$

We will write $\Sigma_{k}$ for the subspace consisting of those polynomials which have a root of multiplicity greater than $k$. This space is obviously of dimension $k$ and it can be shown that the form $\omega$ vanishes on its regular locus. So we have a lagrangian subvariety in the space $\mathcal{P}_{2 k+1}$, which is called open swallowtail. To get a more concrete impression of how it looks like, we will describe the easiest examples. For $k=1, \Sigma_{1} \subset \mathcal{P}_{3}$ is just the ordinary cusp in the plane, this 


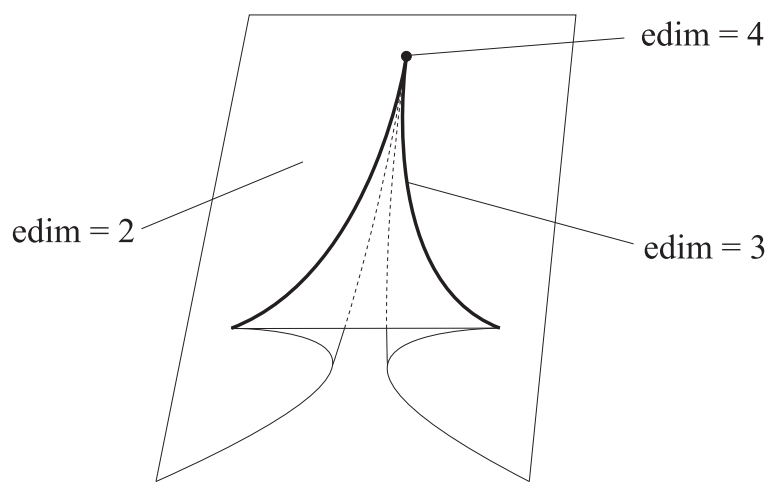

Figure 1: The open swallowtail $\Sigma_{2} \subset \mathbb{K}^{4}$

case has already been discussed above. For $k=2$, we obtain a surface in the four-dimensional space (see the conceptual figure i1)

$$
\mathcal{P}_{5}=\left\{x^{5}+A x^{3}+B x^{2}+C x+D \mid(A, B, C, D) \in \mathbb{K}^{4}\right\}
$$

(the symplectic form is $\omega=3 d A \wedge d D+d C \wedge d B$ ) consisting of those polynomials $f$ with a root of multiplicity at least three. Such a $f$ can be written as $f=$ $(x-a)^{3}\left(x^{2}+3 a x+b\right)$, so there is a normalization of $\Sigma_{2}$ given by

$$
\begin{aligned}
n: \mathbb{K}^{2} & \longrightarrow \mathcal{P}_{5}=\mathbb{K}^{4} \\
(a, b) & \longmapsto\left(b-6 a^{2}, 8 a^{3}-3 a b, 3 a^{2} b-3 a^{4},-a^{3} b\right)
\end{aligned}
$$

Note that the singular locus of $\Sigma_{2}$ is a again a cusp as well as the transversal curve singularity.

The space $\Sigma_{2}$ is our main example, we will describe in some detail how to apply the general results in this case. Using elimination theory, we can calculate the defining equations of $\Sigma_{2}$ in $\mathbb{K}^{4}$. It turns out that the swallowtail is a determinantal variety given by the minors of the matrix

$$
\left(\begin{array}{cc}
9 D & 9 B^{2}-32 A C \\
3 C & -5 A B+125 D \\
-9 B & 45 A^{2}-100 C
\end{array}\right)
$$

The ideal which defines $\Sigma_{2}$ is generated by the following three polynomials

$$
\begin{aligned}
& f_{1}=-27 B^{2} C+96 A C^{2}-45 A B D+1125 D^{2}, \\
& f_{2}=81 B^{3}-288 A B C+405 A^{2} D-900 C D \\
& f_{3}=-45 A B^{2}+135 A^{2} C-300 C^{2}+1125 B D
\end{aligned}
$$

So $\Sigma_{2}$ is not a complete intersection but nevertheless Cohen-Macaulay by the Hilbert-Burch theorem. We list the commutators $\left\{f_{i}, f_{j}\right\}$ (for $1 \leq i<j \leq 3$ ) with respect to the given set of generators (this is a direct proof that $\Sigma_{2} \subset \mathbb{K}^{4}$ is involutive):

$$
\begin{aligned}
& \left\{f_{1}, f_{2}\right\}=-576 A f_{1}+81 B f_{2}-96 C f_{3} \\
& \left\{f_{1}, f_{3}\right\}=15 A f_{2}-12 B f_{3} \\
& \left\{f_{2}, f_{3}\right\}=-900 f_{1}+18 A f_{3}
\end{aligned}
$$


$\Sigma_{2}$ is quasi-homogeneous with the weights $(2,3,4,5)$ for the variables $A, B, C$, $D$, respectively. We can thus apply the machinery developed above to obtain that $\left(\mathcal{L} \mathcal{T}_{\Sigma_{2}}^{1}\right)_{0}=0$, while $\left(\mathcal{L} T_{\Sigma_{2}}^{2}\right)_{0}=\mathbb{K}$. The operator $D$ is in this case

$$
t \partial_{t} \mathbf{1}+\left(\begin{array}{cccc}
11 / 40 & -245 / 2 & 0 & 0 \\
33 / 4000 & 109 / 40 & 0 & 0 \\
0 & 0 & 49 / 15 & -59 / 27 \\
0 & 0 & 51 / 100 & 11 / 15
\end{array}\right)
$$

For $\mathbb{K}=\mathbb{C}$, the monodromy of the locally constant sheaf $\mathcal{L} \mathcal{T}_{\mid \operatorname{Sing}}^{1}\left(\Sigma_{2}\right) \backslash 0$ has the following eigenvalues

$$
-\frac{8}{10},-\frac{13}{10},-\frac{22}{10},-\frac{27}{10}
$$

The second large class of examples are the conormal spaces. Given any submanifold $Y$ of an $n$-dimensional manifold $X$, the total space of the conormal bundle $T_{Y}^{*} X$ is always a lagrangian submanifold of $T^{*} X$. More generally, if $Y$ is an analytic subspace, we can take the closure of the space of conormals to all smooth points of $Y$. The result (which is called conormal space of $Y$ in $X$ ) is still lagrangian, but may have singularities. This is an important class of lagrangian subvarieties, as the characteristic variety of a holonomic $\mathcal{D}_{X}$-module is always a finite union of conormal spaces. Obviously, these spaces are conical in the fibers of $T^{*} X$. If $X$ is a plane curve in $C \subset \mathbb{K}^{2}$, then the conormal space $T_{C}^{*} \mathbb{K}^{2}$ will be a surface in $\mathbb{K}^{4}$. Here the results are as follows.

\begin{tabular}{r|l|l|l} 
equation of $C$ & $\mathbf{L T}^{\mathbf{1}}$ & $\mathbf{L T}^{\mathbf{2}}$ & eigenvalues (multiplicity, if $\neq 1$ ) \\
\hline$y^{2}-x^{5}$ & 0 & 0 & $-\frac{4}{5},-\frac{16}{5}$ \\
\hline$y^{3}-x^{7}$ & 0 & 0 & $-\frac{37}{7},-\frac{61}{7},-\frac{69}{7},-\frac{85}{7},-\frac{93}{7},-\frac{117}{7}$ \\
\hline$y^{5}-x^{7}$ & 0 & 0 & $-\frac{116}{7},-\frac{132}{7},-\frac{148}{7},-\frac{164}{7}$, \\
\hline$y^{3}-x^{6}$ & $\mathbb{K}$ & $\mathbb{K}$ & $-\frac{7}{2},-\frac{10}{2}{ }^{(2)},-\frac{13}{2}$ \\
\hline$x y(x+y)(x-y)(x-2 y)$ & $\mathbb{K}^{2}$ & $\mathbb{K}^{2}$ & -
\end{tabular}

In the last example, there is only an isolated singularity, so the modules $\mathcal{G}_{L}^{1}$ and $\mathcal{G}_{L}^{2}$ are artinien.

Finally, there is a third class of singular lagrangian subvarieties, these are completely integrable hamiltonian systems. Such a system is given in the $2 n$ dimensional phase space by $n$ Poisson-commuting functions. The ideal formed by them then obviously satisfies the involutivity condition. If, additionally, the common zero set of these function is a complete intersection, then it will be lagrangian in our sense. The lagrangian deformation space of such a system is at least $n$-dimensional (addition of a constant is flat and the ideal stays involutive).

To get the equations of some interesting examples, we will proceed as follows. Choose coordinates $\left(p_{1}, q_{1}, p_{2}, q_{2}\right)$ of $\mathbb{K}^{4}$ and set $z_{1}=p_{1}+i q_{1}$ and $z_{2}=p_{2}+i q_{2}$ (This can obviously be done only in the real case, but it is a formal calculus which works as well for $\mathbb{K}=\mathbb{C}$ as for $\mathbb{K}=\mathbb{R}$ ). We can now express functions on $\mathbb{K}^{4}$ in the variables $z_{1}, z_{2}, \overline{z_{1}}, \overline{z_{2}}$, and the Poisson bracket becomes

$$
\{f, g\}=2 i\left(\partial_{\bar{z}_{1}} f \cdot \partial_{z_{1}} g-\partial_{\bar{z}_{1}} g \cdot \partial_{z_{1}} f+\partial_{\bar{z}_{2}} f \cdot \partial_{z_{2}} g-\partial_{\bar{z}_{2}} g \cdot \partial_{z_{2}} f\right)
$$


We want to find functions $f_{1}, f_{2}$ such that $\left\{f_{1}, f_{2}\right\}=0$. Set, for example $f=\lambda z_{1} \overline{z_{1}}+\mu z_{2} \overline{z_{2}}$ and let us look for a $g=z_{1}^{\alpha} \overline{z_{1}} \beta z_{2}^{\gamma} \overline{z_{2}} \delta$ for some parameters $\lambda, \mu, \alpha, \beta, \gamma, \delta \in \mathbb{N}$. It can be easily verified that the commuting condition transforms to

$$
\lambda(\alpha-\beta)-\mu(\gamma-\delta)=0
$$

The following table shows results for some resonance (r) coefficients $\lambda, \mu$ and exponents $(\mathbf{e}) \alpha, \beta, \gamma, \delta$.

\begin{tabular}{r|c|l|l|l}
$\mathbf{r}$ & $\mathbf{e}$ & $\mathbf{L} \mathbf{T}^{\mathbf{1}}$ & $\mathbf{L} \mathbf{T}^{\mathbf{2}}$ & eigenvalues (multiplicity) \\
\hline 1,0 & $0,0,1,1$ & $\mathbb{K}^{2}$ & $\mathbb{K}$ & $-3^{(4)}$ \\
\hline 1,2 & $0,2,1,0$ & $\mathbb{K}^{3}$ & $\mathbb{K}^{2}$ & $-\frac{2}{2}^{(2)},-\frac{3}{2}^{(2)},-\frac{4}{2}^{(2)},-\frac{5}{2}^{(2)},-\frac{6}{2}^{(2)}$ \\
\hline 1,3 & $3,0,0,1$ & $\mathbb{K}^{4}$ & $\mathbb{K}^{3}$ & $-\frac{3}{3}^{(2)},-\frac{5}{3}^{(2)},-\frac{7}{3}^{(4)},-\frac{9}{3}^{(4)},-\frac{11}{3}^{(4)},-\frac{13}{3}^{(2)},-\frac{15}{3}^{(2)}$ \\
\hline 1,4 & $4,0,0,1$ & $\mathbb{K}^{5}$ & $\mathbb{K}^{4}$ & $\begin{array}{l}-\frac{4}{4}^{(2)},-\frac{7}{4}^{(2)},-\frac{9}{4}^{(2)},-\frac{10}{4}^{(2)},-\frac{12}{4}^{(2)},-\frac{13}{4}^{(2)}, \\
-\frac{14}{4}^{(2)},-\frac{15}{4}^{(2)},-\frac{16}{4}^{(2)},-\frac{17}{4}^{(2)},-\frac{18}{4}^{(2)},-\frac{19}{4}^{(2)}, \\
-\frac{20}{4}^{(2)},-\frac{22}{4}^{(2)},-\frac{23}{4}^{(2)},-\frac{25}{4}^{(2)},-\frac{28}{4}^{(2)}\end{array}$
\end{tabular}

Remark: The eigenvalues in all examples have a symmetry property, which we cannot prove at this moment. These eigenvalues looks very similar to the spectrum of an isolated hypersurface singularity. One might speculate that there is a mixed Hodge structure related to this theory and that the eigenvalues share further properties with the spectrum, e.g. the semi-continuity under deformations.

\section{References}

[Ban94] A. Banyaga, An introduction to symplectic geometry, Progress in Mathematics, vol. 117, Birkhaeuser, 1994, pp. 17-40.

[BG80] Ragnar-Olaf Buchweitz and Gert-Martin Greuel, The Milnor number and deformations of complex curve singularities, Invent. Math. 58 (1980), 241-281.

[Bjö93] Jan-Erik Björk, Analytic D-modules and applications, Mathematics and its Applications, vol. 247, Kluwer Academic Publishers, 1993.

[CJNMM96] Francisco J. Castro-Jiménez, Luis Narváez-Macarro, and David Mond, Cohomology of the complement of a free divisor, Trans. Am. Math. Soc. 348 (1996), no. 8, 3037-3049.

[Dou74] Adrien Douady, Le théorème des images directes de Grauert (d'après Kiehl-Verdier), Asterisque 16 (1974), 49-62.

[Giv82] A.B. Givental', Varieties of polynomials having a root of fixed comultiplicity, and the generalized Newton equation, Funct. Anal. Appl. 16 (1982), 10-14. 
[Giv83] A.B. Givental', Lagrangian manifolds with singularities and irreducible sl $l_{2}$-modules, Russ. Math. Surv. 38 (1983), no. 6, 121-122.

[Giv88] A.B. Givental', Singular Lagrangian manifolds and their Lagrangian mappings, Itogi Nauki Tekh., Ser. Sovrem. Probl. Mat., Novejshie Dostizh. 33 (1988), 55-112, English translation: J. Soviet Math. 52.4 (1988), 3246-3278.

[KV71] Reinhardt Kiehl and Jean-Louis Verdier, Ein einfacher Beweis des Kohärenzsatzes von Grauert, Math. Ann. 195 (1971), 24-50.

[Mac87] K. Mackenzie, Lie groupoids and Lie algebroids in differential geometry, London Mathematical Society Lecture Note, vol. 124, Cambridge University Press, 1987.

[Mal74] Bernard Malgrange, Intégrales asymptotiques et monodromie, Ann. Sci. Ec. Norm. Super., IV. Ser. 7 (1974), 405-430.

[Mat] Daisuke Matsushita, Equidimensionality of complex lagrangian fibrations, preprint alg-geom/9911166.

[Mat89] Hideyuki Matsumura, Commutative ring theory, Cambridge Studies in Advanced Mathematics, vol. 8, Cambridge University Press, 1989 .

[Sch68] M. Schlessinger, Functors of Artin rings, Trans. Am. Math. Soc. 130 (1968), 208-222.

[Sev99] Christian Sevenheck, Lagrange-Singularitäten und ihre Deformationen, Diploma thesis, Heinrich-Heine-Universität Düsseldorf (1999).

[Voi92] Claire Voisin, Sur la stabilité des sous-variétés lagrangiennes des variétés symplectiques holomorphes, Lect. Note, vol. 179, Lond Math. Soc., 1992, pp. 294-303.

[vS87] D. van Straten, On the Betti numbers of the Milnor fibre of a certain class of hypersurface singularities, Singularities, representation of algebras, and vector bundles (Gert-Martin Greuel and Günter Trautmann, ed.), Lect. Notes Math., vol. 1273, SpringerVerlag, 1987, pp. 203-220.

[Wei90] Alan Weinstein, Connections of Berry and Hannay type for moving Lagrangian submanifolds, Adv. Math. 82 (1990), no. 2, 133-159 (English).

FB 17, Mathematik,

Johannes-Gutenberg-Universität Mainz, D-55099 Mainz, Germany

straten@mathematik.uni-mainz.de, sevenhec@mathematik.uni-mainz.de 